\title{
Intestinal proteome changes during infant necrotizing enterocolitis
}

\author{
Pingping Jiang ${ }^{1,2}$, Birgitte Smith ${ }^{3}$, Niels Qvist ${ }^{4}$, Christian Nielsen ${ }^{5}$, Jennifer Man-Fan Wan ${ }^{1}$, Wai-Hung Sit ${ }^{1}$, Tim Kåre Jensen ${ }^{6}$,
} Hualin Wang ${ }^{1}$ and Per Torp Sangild ${ }^{2}$

BACKGROUND: Changes in the intestinal and colonic proteome in patients with necrotizing enterocolitis (NEC) may help to characterize the disease pathology and identify new biomarkers and treatment targets for NEC.

METHODS: Using gel-based proteomics, proteins in NECaffected intestinal and colonic sections were compared with those in adjacent, near-normal tissue sections within the same patients. Western blot and immunohistochemistry were applied to crossvalidate proteomic data and histological location of some selected proteins.

RESULTS: Thirty proteins were identified with differential expression between necrotic and vital small-intestine sections and 23 proteins were identified for colon sections. Five proteins were similarly affected in the small intestine and colon: histamine receptors (HRs), actins, globins, immunoglobulin, and antitrypsin. Two heat shock proteins (HSPs) were affected in the small intestine. Furthermore, proteins involved in antioxidation, angiogenesis, cytoskeleton formation, and metabolism were affected. Finally, secretory proteins such as antitrypsin, fatty-acid binding protein 5, and haptoglobin differed between NEC-affected and vital tissues.

CONCLUSION: NEC progression affects different pathways in the small intestine and colon. HSPs may play an important role, especially in the small intestine. The identified secretory proteins should be investigated as possible circulating markers of NEC progression in different gut regions.

N ecrotizing enterocolitis (NEC) is the most severe gastrointestinal complication in preterm infants. Although the clinical features of NEC are well known, the pathogenesis in the tissue remains unclear (1), thereby hindering effective preventive and therapeutic interventions. The current understanding of NEC includes intolerance to enteral feeding, leading to nutrient fermentation, bacterial overgrowth, and toxin production, which subsequently trigger a tissue inflammatory response, mucosal atrophy, and transmural necrosis. It continues to be debated whether factors such as tissue ischemia and the associated reperfusion injury contribute to NEC, and particularly whether they are early predisposing factors or late consequences of the disease progression (2). The distal ileum and proximal colon are the most frequently affected regions, but sometimes NEC affects all regions of the gastrointestinal tract from the stomach to the rectum (2). It is believed that tissue lesions advance rapidly, over a few days, from focal patchy sites during the early stages of disease, but the exact time course of NEC remains unclear because access to tissue from mildly affected infants is limited.

No effective prevention strategy for NEC is known so far, although slow advancement of enteral food, particularly mother's milk, has been documented to reduce NEC incidence (3). Clinical signs (e.g., lethargy, abdominal distension and discoloration, food intolerance, intestinalis pneumatosis) lead to different forms of interventions (e.g., enteral food restriction, antibiotics, intestinal resection, peritoneal drainage) that differ among hospitals. There is a need to understand the key elements of NEC progression, both locally in the gut and at the whole-body level.

Animal models of NEC may help to identify the crucial steps in disease pathology, but it remains a large challenge to translate the results from rodent and pig models to human infants. In a large proportion of pigs, preterm birth and formula feeding induce spontaneous development of intestinal lesions, which is very similar to infant NEC $(4,5)$, without the need for artificial insults such as the hypoxia, hypothermia, bacterial challenge, or aggressive formula feeding often used in rodent NEC models. A series of recent studies investigated the intestinal proteome in preterm pigs during the progression of feeding- and bacteriainduced NEC (6-8). Proteomics allows simultaneous detection of hundreds of proteins, and we showed that the key cellular and physiological processes that characterized NEC development included intestinal stress response, protein processing and degradation, metabolism of selected amino acids (arginine, ornithine), carbohydrates, and energy (6-8). Similar studies are difficult to perform in infants, but the results from pigs may help to understand proteome differences between healthy and NECaffected parts of the infant gut.

By gel-based proteomics, proteins are separated based on their $\mathrm{pI}$ and $\mathrm{M}_{\mathrm{r}}$ values, and the fluorescent staining dye provides a signal proportional to the protein amount. Protein identification based on the mass spectra allows reliable detection of

\footnotetext{
'School of Biological Sciences, University of Hong Kong, Hong Kong, China; ${ }^{2}$ Department of Human Nutrition, Faculty of Science, University of Copenhagen, Frederiksberg, Denmark; ${ }^{3}$ Department of Microbiological Surveillance and Research, State Serum Institute, Copenhagen, Denmark; ${ }^{4}$ Department of Surgery, Odense University Hospital, Odense, Denmark; ${ }^{5}$ Department of Clinical Immunology, Hans Christian Andersen Children's Hospital and Odense University Hospital, Odense, Denmark; ${ }^{6}$ National Veterinary Institute, Technical University of Denmark, Copenhagen, Denmark. Correspondence: Per Torp Sangild (psa@life.ku.dk)
} 
Table 1. Clinical information of patients included for proteomic analyses

\begin{tabular}{|c|c|c|c|c|c|c|c|c|c|}
\hline ID & $\begin{array}{l}\text { Gestation } \\
\text { age }(d)\end{array}$ & $\begin{array}{l}\text { Birth } \\
\text { weight (g) }\end{array}$ & Delivery & $\begin{array}{c}\text { Age at } \\
\text { surgery (d) }\end{array}$ & $\begin{array}{c}\text { Bell's } \\
\text { classification }\end{array}$ & Intestinal pathology & Feeding & Peritonitis & Outcome \\
\hline 1 & $30+5$ & 1,060 & $\begin{array}{l}\text { Cesarean } \\
\text { section }\end{array}$ & 1 & IIIB & $\begin{array}{l}\text { Gangrene in ileum } \\
(10 \mathrm{~cm}) \text { with perforation }\end{array}$ & None & Fecal diffuse & Survived \\
\hline 3 & $24+5$ & 664 & $\begin{array}{l}\text { Cesarean } \\
\text { section }\end{array}$ & 46 & $\| A$ & Gangrene in colon & $\begin{array}{l}\text { Mother's } \\
\text { milk + PN }\end{array}$ & None & $\begin{array}{l}\text { Died (cvk to } \\
\text { mediastinum) }\end{array}$ \\
\hline 5 & $32+0$ & 1,170 & $\begin{array}{l}\text { Cesarean } \\
\text { section }\end{array}$ & 4 & IIIA & Gangrene in right colon & PN & $\begin{array}{l}\text { Ascites and } \\
\text { fibrin }\end{array}$ & Survived \\
\hline 6 & $34+1$ & 2,110 & Vaginal & 13 & IIB & Gangrene in colon & $\begin{array}{l}\text { Mother's } \\
\text { milk + PN }\end{array}$ & $\begin{array}{l}\text { Ascites and } \\
\text { fibrin }\end{array}$ & Survived \\
\hline 9 & $32+6$ & 2,260 & Vaginal & 22 & IIIB & $\begin{array}{l}\text { Necrosis and perforation } \\
\text { in distal ileum }\end{array}$ & $\begin{array}{l}\text { Mother's } \\
\text { milk }\end{array}$ & Fecal diffuse & Survived \\
\hline 10 & $32+5$ & 2,286 & $\begin{array}{l}\text { Cesarean } \\
\text { section }\end{array}$ & 31 & IV & $\begin{array}{l}\text { Necrosis and perforation } \\
\text { in distal ileum }\end{array}$ & $\begin{array}{l}\text { Mother's } \\
\text { milk + PN }\end{array}$ & Fecal diffuse & Died \\
\hline
\end{tabular}

cvk, central venous catheter; PN, parenteral nutrition.

specific proteins. Only proteins with extreme $\mathrm{pI}$ or $\mathrm{M}_{\mathrm{r}}$, or those present with low abundance, are difficult to separate, identify, and quantify with gel-based proteomics. More sensitive liquid chromatography-based proteomic approaches are needed to detect such proteins.

We hypothesized that the tissue proteome differences between NEC-affected and adjacent near-normal intestinal sections from the same infant would be similar to the differences observed previously between NEC and healthy pigs. Furthermore, we speculate that the NEC-affected tissue responses differ between the colon and small intestine, due to the marked differences in structure and functions of these two gastrointestinal regions. Using methods identical to those used in the pig experiments (6-8), we aimed to better characterize NEC progression in infants and some proteins that are central in the disease process and might be relevant targets for biomarker development or NEC therapy.

\section{RESULTS}

\section{Clinical Characteristics and Histology Findings}

Ten neonates with different gestation ages were enrolled in this study. Clinical characteristics are described in Table 1, and representative histological sections of necrotic and vital sections from the small intestine and colon are shown in Figure 1. The vital small-intestinal sections appeared normal or showed mild local disruption of villi but never separation of submucosal layers or serious hemorrhage (Figure 1a). Conversely, the NEC-affected small-intestinal sections showed extensive destruction of the villus architecture, separation of submucosal layers, and hemorrhage (Figure 1b). Likewise, in colon sections, only mild disruption of the crypt structure, without any hemorrhage or separation of the submucosal layers was present in vital sections (Figure 1c), as compared with NECaffected colonic sections (Figure 1d).

\section{Proteomics}

The molecular weight range of the proteins separated on twodimensional gel electrophoresis (2-DE) gel was $15-250 \mathrm{kDa}$ and $~ 500$ spots could be detected on the gels by the analyzing software PDQuest (Bio-Rad, Hercules, CA). For the small intestine, 30 proteins were identified with significantly different expression levels in NEC-affected vs. vital sections, whereas 23 proteins were identified in the colon sections. The positions of these identified proteins on the two-dimensional gel electrophoresis gels are shown in Figure 2 (NEC-affected and vital small intestine (Figure 2a,b); NEC-affected and vital colon (Figure 2c,d)). Descriptive information of the identified proteins, including spot number, protein name, protein score, and expression quantity in each group (NEC-affected and vital), are listed in Tables 2 and 3.

Five protein groups showed changes in both the small-intestine and colon regions, and these proteins were histamine receptor (HR) subunit peptide 1 (spot 1012 in Tables 2 and 3), $\alpha$ actin (spot 1006, 2411 in Table 2 and spot 2411 in Table 3), immunoglobin J chain (spot 0122 in Table 2, spot 0121 and 0122 in Table 3), and g- $\gamma$ globin (spot 6005, 6007 in Table 2 and spot 6007, 7003 in Table 3). Other cytoskeletal proteins affected by NEC were $\beta$-actin (spot 0017), $\alpha 2$ actin (spot 1006), and keratin 8 (spot 3607) in the small intestine, and $\gamma 2$ actin (spot 0409) and keratin 6A (spot 3505) in the 

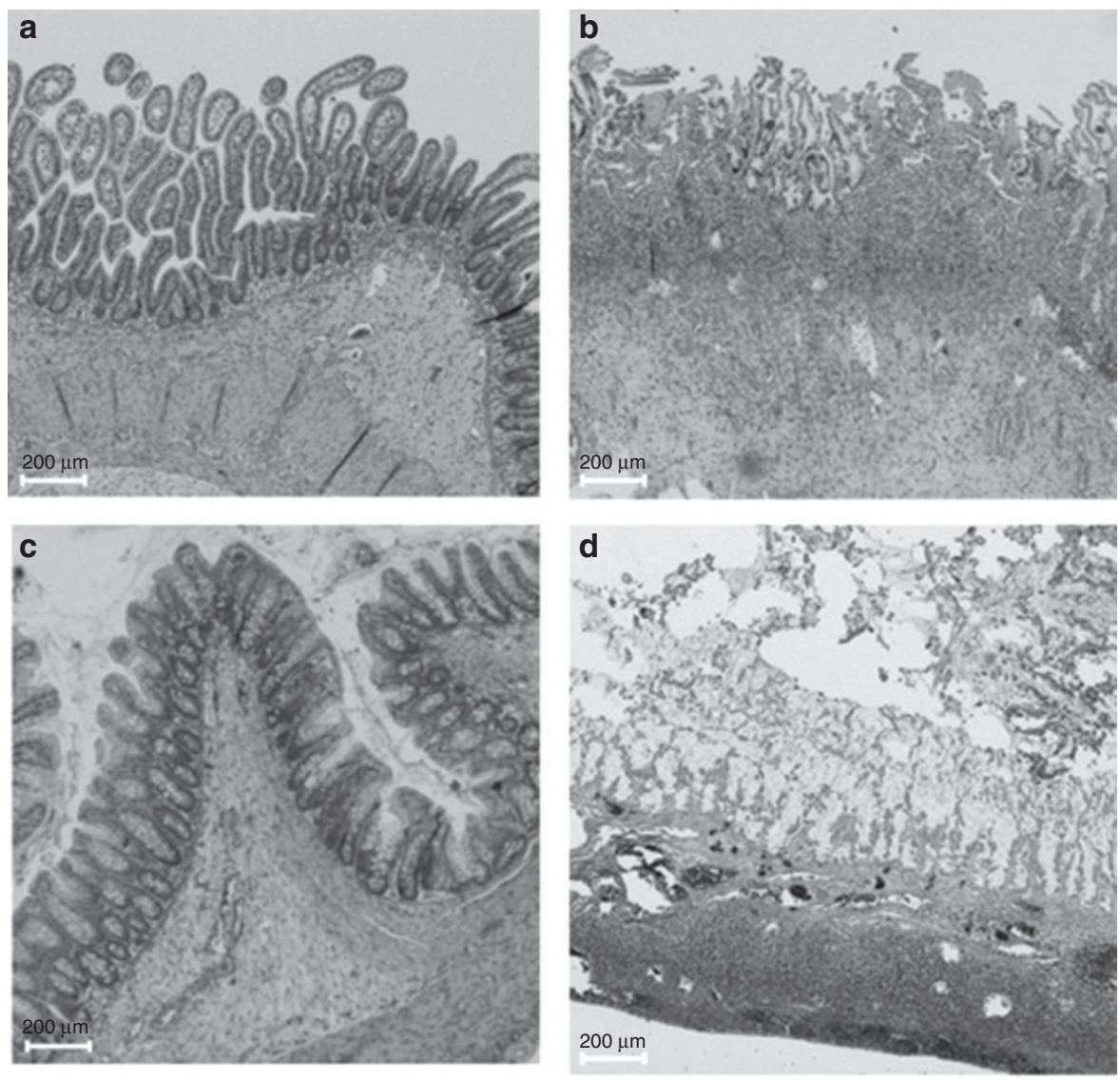

Figure 1. Representative histological pictures of vital and NEC-affected small intestine and colon (original magnification $\times 50$ ). (a) Vital small intestine: mild local disruption of villi, no separation of submucosal layers; (b) NEC-affected small intestine: extensive destruction of mucosal structure, blood congestion; (c) vital colon: mild disruption of villi structure, no separation of submucosal layers; (d) NEC-affected colon: thorough disruption of mucosal layer, separation of submucosal layers and blood congestion. NEC, necrotizing enterocolitis.

colon. A number of other proteins related to the cytoskeleton and cell-cell and cell-matrix junctions also showed differences as a result of NEC progression in the small intestine (cytovillin 2, spot 4601; motor protein, spots 4805 and 4810; vinculin, spot 5906; septin 11, spot 6405; Table 2).

Two heat shock proteins (HSPs), HSPA5 and HSP27, were identified with NEC-related differential expression in the small intestine (spots 0705 and 3110, respectively, Table 2). Likewise, fatty-acid binding protein 5 , a well-known marker of intestinal inflammation, was downregulated in necrotic small intestine, and lactoferrin (spot 4309) and proteins related to antioxidation, such as heme-binding protein 2 (spot 0113) and biliverdin reductase B (spot 8004) were also affected (Table 2); in the colon, thioredoxin-like 1 (spot 0206) and peroxiredoxin 1 (spot 7006) were affected by NEC progression (Table 3 ).

Numerous proteins related to carbohydrate and protein metabolism were affected by NEC progression, including ketohexokinase isoform b (spot 4204), aldehyde dehydrogenase (spot 6515), and pyruvate kinase (spot 8603) in the small intestine (Table 2), and ubiquinol-cytochrome $c$ reductase (spot 6102), pyruvate dehydrogenase (spot 6305), and carbonic anhydrase (spot 7106) in the colon (Table 3). Propionyl-coenzyme A carboxylase a polypeptide (spot 5706) and 5-aminoimidazole-4-carboxamide ribonucleotide formyltransferase/inosine monophosphate cyclohydrolase bifunctional enzyme (spot 6613) were identified in the small intestine (Table 2), whereas HMGCS2 (spot 7401) was observed in the colon (Table 3). As indicated above, these protein-expression changes were region specific and had no consistent direction of change (up or down), reflecting highly complex and site-specific changes in cell metabolism during NEC progression.

\section{Western Blot and Immunohistochemistry of HSPA5 and HSP27}

The relative abundance of two selected proteins, HSPA5 and HSP27, was crossvalidated by western blot (Figure 3a,c). HSPA5 showed lower levels in NEC-affected intestines than in the vital tissues $(P<0.05)$, whereas HSP27 showed higher levels in NECaffected intestine. The localization of HSPA5 and HSP27 in the small intestine was documented by immunohistochemistry using specific antibodies. The immunostaining against these two proteins showed that they were mainly expressed in the outer layer of intestinal villi where the enterocytes were in contact with the luminal content (digesta, endogenous secretions, and bacteria; Figure 3b,d).

\section{DISCUSSION}

The difficulties in obtaining human NEC intestinal specimens and corresponding control tissue for biochemical 

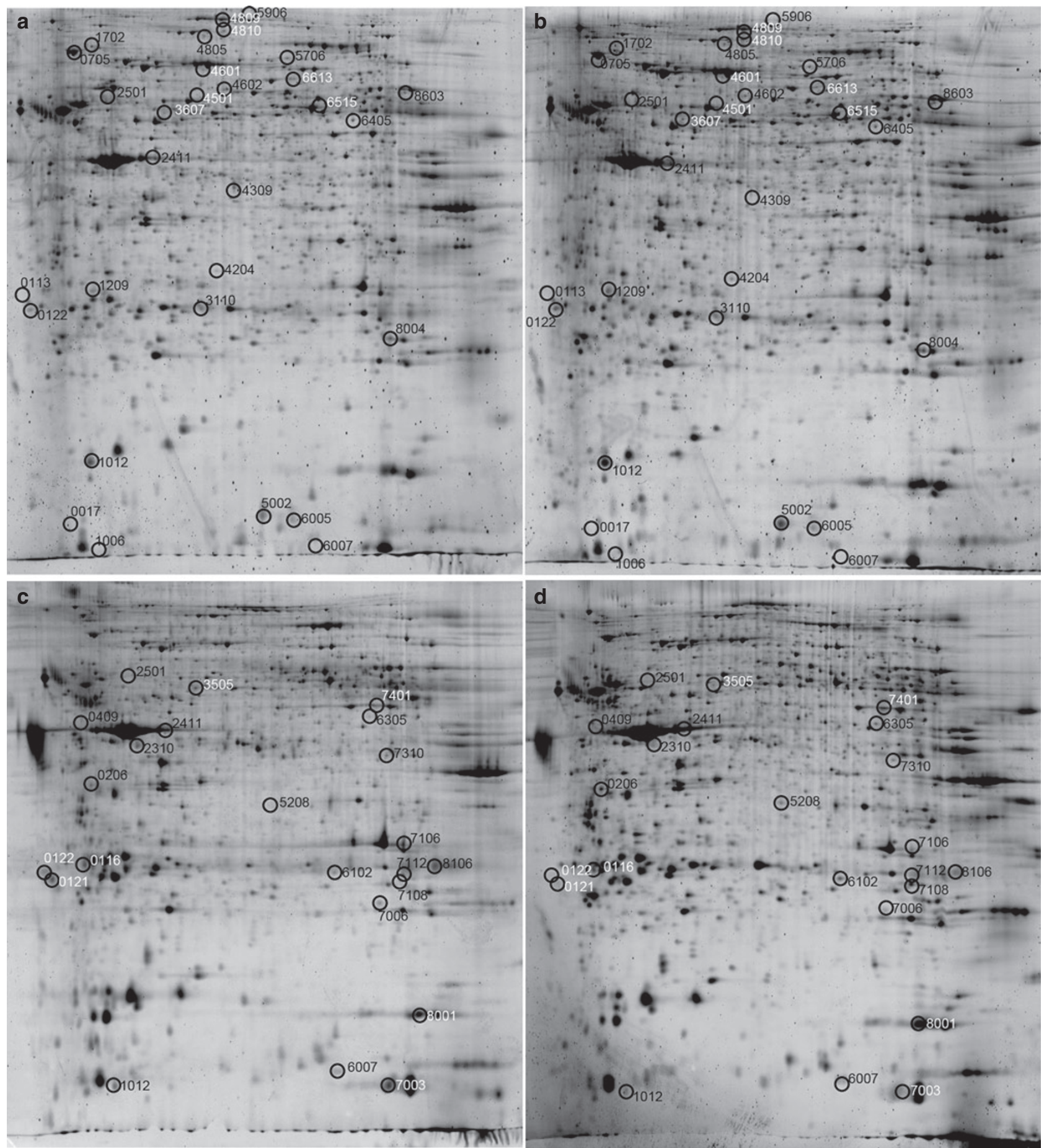

Figure 2. Representative 2-DE gel graph of NEC-affected and vital $(\mathbf{a}, \mathbf{b})$ small intestine and $(\mathbf{c}, \mathbf{d})$ colon. All spots circled and labeled with a number represent protein spot identified; the numbers labeled here are consistent with ones listed in Tables 2 and 3. NEC, necrotizing enterocolitis; 2-DE, twodimensional gel electrophoresis.

analyses have limited the understanding of early and late events of NEC in human infants. For ethical reasons, control intestinal tissue from healthy infants is difficult to obtain, and the quality of tissue removed from infants with other early intestinal complications (e.g., atresia, stenosis, and Hirschsprung's disease) is limited by tremendous differences in the important and confounding factors such as pre- and postnatal age, intestinal region, feeding and antibiotic regimens, genetics, and the associated diseases. We therefore chose to compare the NEC-affected parts of intestines and colon with near-normal vital sections removed from the same infant, which is part of the standard practice for intestinal resection as treatment for NEC. Given that the vital sections removed were adjacent to the necrotic sections, the 


\section{Articles | jianget al.}

Table 2. Identified proteins with differential expression between vital and necrotizing enterocolitis-affected small intestines $(n=6, P<0.05)$

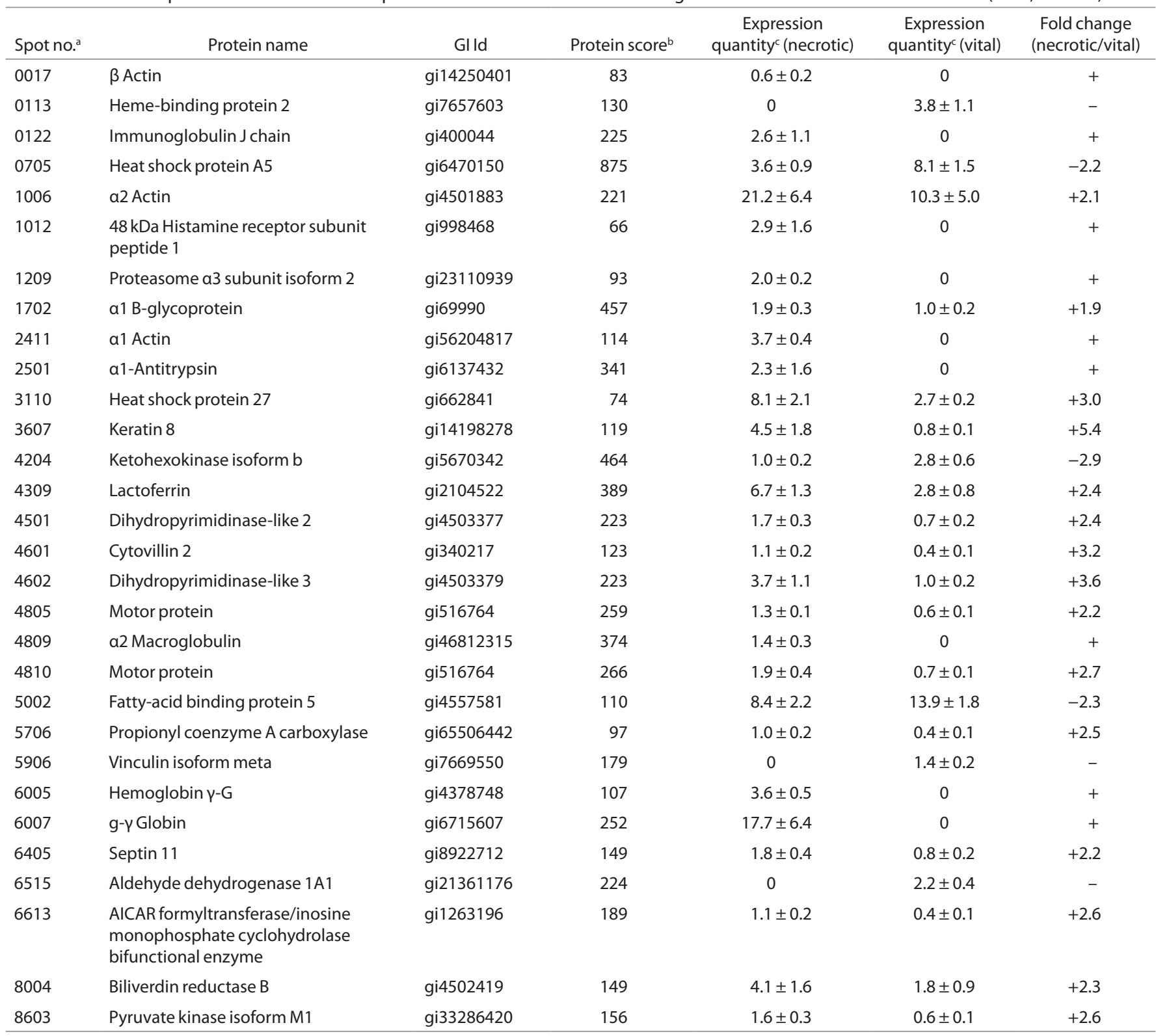

AICAR, 5-aminoimidazole-4-carboxamide ribonucleotide; Gl Id, Genbank identifier.

aspot number: the position of the spots on two-dimensional gel electrophoresis gels as shown in Figure $\mathbf{2}$. ${ }^{\circ}$ Protein score: confidence of identification; the higher the score, the less probable that the identification is false $(P<0.05$ for protein scores $>66)$. ${ }^{\circ}$ Expression quantity: mean expression quantity expressed as the sum of optical density $(O D)$ of the each pixel of spot area $\left(\Sigma \mathrm{OD} \times 10^{5}\right)$.

vital sections were mildly affected and the histological pictures of these vital sections indicated a mild disruption of intestinal structure (Figure 1a,c). Therefore, the proteome changes between vital and NEC-affected tissues in this study provide information mainly about the changes during the late stage of NEC.

We observed in our study that the small intestine and colon showed marked differences in their responses to NEC progression. Only five protein groups were affected in both regions: HRs, actins, globins, immunoglobulin, and a1-antitrypsin. The elevated expression of HRs in NECaffected tissues (both small intestine and colon) may reflect an effect of NEC on the histamine regulation of innate and acquired immune responses and a shift in the systemic Th (helper $\mathrm{T}$ cell) $1 / \mathrm{Th} 2$ balance from Th1 to Th2 to a more allergic state (9). HRs also regulate smooth muscle contraction, gastric acid secretion, and neurotransmitter release (10), indicating that the progression of NEC lesions affects these important functions via increased HR abundance. Similarly, a region-independent marked increase of immunoglobulin production in local tissue was found, which suggests that the progression of NEC is probably associated with invading gut microbes. Increases in g- $\gamma$ globin (a hemoglobin) in both regions may reflect a high need for oxygen in this crucial process but could also reflect hemorrhage. In addition, the increased local expression of a1-antitrypsin found in both 
Table 3. Identified proteins with differential expression between vital and necrotic colon $(n=6, P<0.05)$

\begin{tabular}{|c|c|c|c|c|c|c|}
\hline Spot no. ${ }^{a}$ & Protein name & Gl Id & Protein score ${ }^{b}$ & $\begin{array}{l}\text { Expression quantity } \\
{\text { (necrotic })^{c}}\end{array}$ & $\begin{array}{c}\text { Expression } \\
\text { quantity }(\text { vital) }\end{array}$ & $\begin{array}{l}\text { Fold change } \\
\text { (necrotic/vital) }\end{array}$ \\
\hline 0116 & Rho GDP dissociation inhibitor 1 & gi4757768 & 76 & $4.8 \pm 0.8$ & $2.4 \pm 0.4$ & +2.0 \\
\hline 0121 & Immunoglobulin J chain & gi21489959 & 194 & $9.4 \pm 3.1$ & $1.9 \pm 0.5$ & +5.0 \\
\hline 0122 & Immunoglobulin J chain & gi400044 & 225 & $7.0 \pm 2.1$ & 0 & + \\
\hline 0206 & Thioredoxin-like 1 & gi4759274 & 217 & $1.5 \pm 0.3$ & $3.2 \pm 0.8$ & -2.2 \\
\hline 0409 & ү2 Actin & gi49168516 & 102 & 0 & $1.2 \pm 0.4$ & - \\
\hline 2310 & Haptoglobin protein & gi47124562 & 128 & $4.6 \pm 0.8$ & $2.1 \pm 0.4$ & +2.2 \\
\hline 2411 & a1 Actin & gi56204817 & 114 & $5.9 \pm 1.0$ & 0 & + \\
\hline 2501 & a1-Antitrypsin & gi6137432 & 341 & $1.7 \pm 0.3$ & $0.8 \pm 0.2$ & +2.0 \\
\hline 3505 & Keratin 6a & gi46812692 & 333 & $1.9 \pm 0.2$ & $0.9 \pm 0.0$ & +2.2 \\
\hline 6305 & Pyruvate dehydrogenase a1 precursor & gi4505685 & 330 & $0.7 \pm 0.1$ & $1.5 \pm 0.3$ & -2.0 \\
\hline 7003 & $g-\gamma-$ Globin & gi6715607 & 470 & $77.5 \pm 24.7$ & $23.9 \pm 9.5$ & +3.2 \\
\hline 7006 & Peroxiredoxin 1 & gi55959887 & 116 & $3.9 \pm 0.9$ & $2.0 \pm 0.4$ & +1.9 \\
\hline 7106 & Carbonic anhydrase II & gi9257153 & 222 & $10.6 \pm 1.2$ & $2.2 \pm 0.5$ & +4.9 \\
\hline 7108 & Karyopherin & gi5107637 & 291 & $2.5 \pm 0.6$ & $4.9 \pm 0.7$ & -1.9 \\
\hline 7112 & Agglutinin & gi10835792 & 217 & $23.2 \pm 5.4$ & $10.2 \pm 4.0$ & +2.3 \\
\hline 7310 & Arginase & gi178995 & 156 & $1.9 \pm 0.4$ & $0.8 \pm 0.2$ & +2.3 \\
\hline 7401 & $\begin{array}{l}\text { 3-Hydroxy-3-methylglutaryl-coenzyme } \\
\text { A synthase } 2\end{array}$ & gi48145817 & 182 & $2.1 \pm 0.5$ & $6.1 \pm 1.0$ & -2.9 \\
\hline 8001 & Chain a, cyclophilin a & gi1633054 & 239 & $16.8 \pm 3.3$ & $34.2 \pm 6.4$ & -2.0 \\
\hline
\end{tabular}

Gl Id, Genbank identifier.

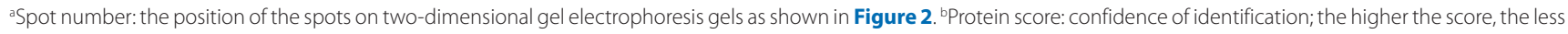
probable that the identification is false $(P<0.05$ for protein scores $>66)$. ${ }^{\circ}$ Expression quantity: mean expression quantity expressed as the sum of optical density (OD) of the each pixel of spot area $\left(\Sigma \mathrm{OD} \times 10^{5}\right)$.

small intestine and colon could protect the intestinal mucosa from the ongoing inflammation damage, similar to its role in inflammatory bowel diseases (11).

Two HSPs (HSPA5 and HSP27) were affected by the progression of NEC lesions in the small intestine as detected by proteomic analysis. No differences were found in the colon sections. Generally, levels of HSPs are regulated by inflammation and metabolic stress, such as that occurring from ischemia reperfusion and oxidative stress. HSPs facilitate the synthesis, folding, distribution, and degradation of many cytoplasmic proteins, and the renaturation or removal of damaged proteins (12). Our previous studies in preterm pigs indicate that the presence of gut microbiota plays a major role in HSPA5 and HSP27 regulation in association with NEC (8). However, the roles of these proteins could be less pronounced in the progression of NEC in the infant intestine, and the upregulation of HSP27 levels detected by proteomics could not be confirmed conclusively by our follow-up western blot analyses. The finding that the two HSPs were detected in greatest abundance in the upper part of the villi in the preterm infant intestine support the notion that luminal factors play an important role in NEC-induced HSP regulation. Here, bacteria-mediated regulation of HSPA5 and HSP27 in NEC tissues may involve the nuclear factor- $\kappa B$ inflammatory pathway by its interaction with the inhibitor of $\kappa B$ kinase complex $(13,14)$. HSP27 also plays an important role in the Ras homolog gene family, member $\mathrm{A}$, and protein kinase C-a pathways (15) that are involved in smooth muscle contraction and epithelial tight junctions.

NEC lesions were associated with an adaptive antioxidative response in which the production of proteins protecting against oxidative stress was altered. The responses differed between the small intestine and colon with regard to the nature and direction (upregulated or downregulated) of affected proteins. Heme-binding proteins bind free heme and thereby help to avoid the production of toxic reactive oxygen species (16). Likewise, the upregulation of biliverdin reductase B in intestinal necrotic tissues serves to protect against oxidative stress and lipid peroxidation via a bilirubin-biliverdin antioxidation cycle (17). The bilirubin-biliverdin antioxidation cycle is known to protect the mucosal architecture following ischemia-reperfusion injury (18), probably via biliverdin reductase working as a kinase to phosphorylate inhibitor of $\kappa \mathrm{B}$, which then inhibits the relocation of nuclear factor- $\mathrm{kB}$ in this inflammatory pathway (18). The observation that thioredoxin-like 1 
a

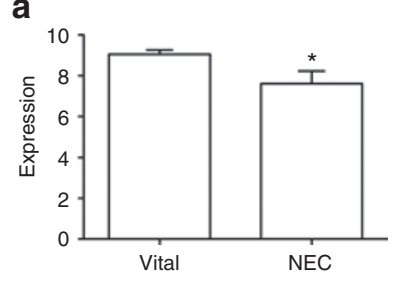

C

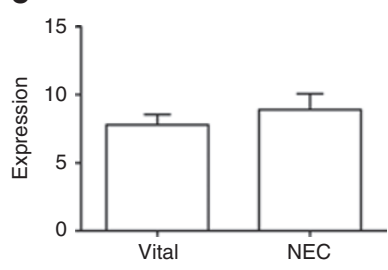

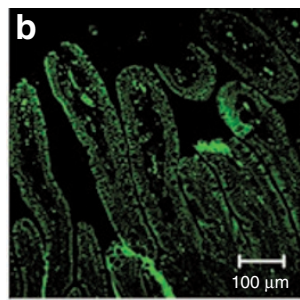
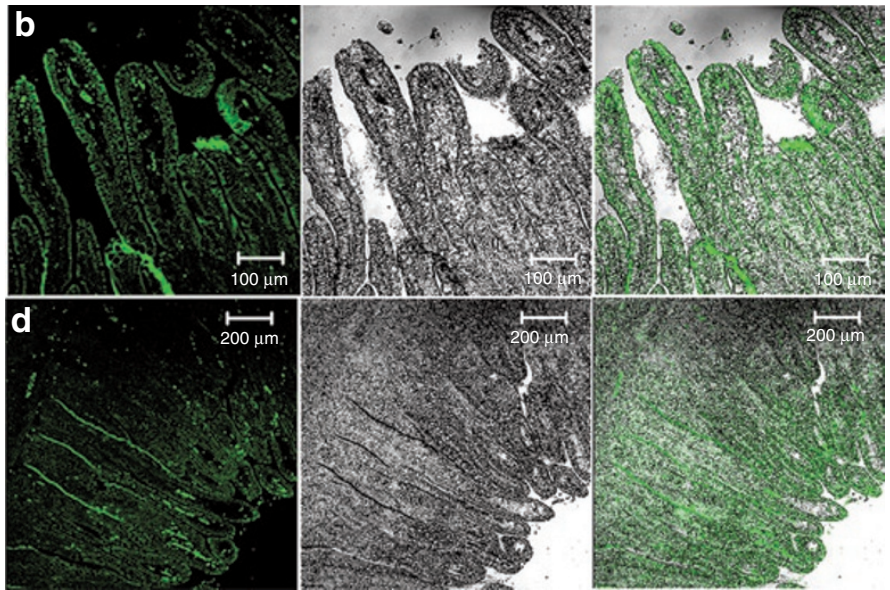

Figure 3. Western blots of (a) HSPA5 and (c) HSP27, and representative immunohistochemical images (original magnification $\times 200$ ) of (b) HSPA5 and (d) HSP27 showing their location in the small-intestine sections with the fluorescein isothiocyanate, bright field, and merged images (from left to right, respectively). The immunohistochemical pictures demonstrate that the HSPA5 and HSP27 proteins are mainly expressed in the outer layer of the intesti-

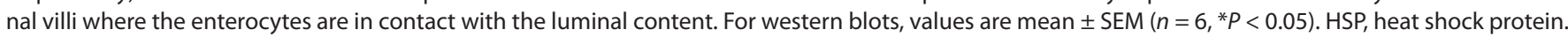

was decreased in necrotic colonic tissue may reflect a reduced metabolic rate (19) and may lead to reduced protection against glucose deprivation-induced cytotoxicity and lowered protein reduction and proteolysis (20). On the other hand, colonic peroxiredoxin 1 was upregulated, thus increasing the cellular protection against reactive oxygen species damage $(21,22)$. This protein is central to eliminating $\mathrm{H}_{2} \mathrm{O}_{2}$ generated by a variety of cellular stresses and proinflammatory cytokines, and it may have a protective role against apoptosis signal-regulating kinase 1-induced apoptosis (23).

As the necrosis advances during NEC, the cytoskeleton proteins probably undergo tremendous change in expression in an attempt to maintain cell integrity. The activation of the cytovillin2 (ezrin)-readixin-moesin family results in tight junction and adherent junction disassembly (24), which is found in NEC. Vinculin is also related to the loss of focal adhesion (25). In addition, the expression of cytovillin 2 is also related to a stress-activated c-Jun $\mathrm{N}$-terminal kinase (24). Keratin 8, a major intermediate filament protein, acts as an antiapoptotic factor in hepatocytes and small intestine (26). Together, effects on the above proteins by NEC progression suggest that enterocyte cytoskeleton rearrangement may be regulated not only by external signals such as attachment of gut microbes but also by intracellular pathways such as the mitogen-activated protein kinases pathway.

The progressing inflammatory lesions changed various metabolic pathways, including lowered metabolism of fructose (ketohexokinase isoform b) (27) and alcohol (aldehyde dehydrogenase) in the small intestine. The higher level of pyruvate kinase in NEC-affected small intestine and lower level of pyruvate dehydrogenase in NEC-affected colon indicate effects on pyruvate metabolism in NEC tissues. Increased metabolism was indicated for pyrimidine and purine (dihydropyrimidase, 5-aminoimidazole-4-carboxamide ribonucleotide formyltransferase/inosine monophosphate cyclohydrolase) and cholesterol (propionyl-Co enzyme A) (28) in intestinal NEC tissues, whereas some differentially regulated proteins in colon suggest NEC-reduced ketogenesis (3-hydroxy-3-methylglutaryl-coenzymeA synthase 2) (29) and increased mitochondrial respiratory chain activity (ubiquinol-cytochrome $c$ reductase) (30).

Plasma concentrations of the intestinal fatty-acid binding protein 2 are elevated in patients with NEC (31), and this may relate to our observation that intestinal fatty-acid binding protein 5 expression was reduced in NEC intestines. The role of this protein remains unclear apart from its known role in fattyacid absorption and transport and in local nervous system and regulation of water permeability (32). A protein with increased intestinal expression in NEC, a1 B-glycoprotein, is elevated in pancreatic juice from patients with pancreatic ductal adenocarcinoma and in hepatocellular carcinoma cell lines and liver tumor tissue samples $(33,34)$. Similarly, the marked expression increase in local intestinal and colonic immunoglobulin production might result in elevated circulating levels of the same components. Finally, increased local colonic production of haptoglobin may give rise to elevated levels of this important circulating acute-phase protein mainly synthesized in hepatocytes in response to infection or inflammation (35). Of note, a precursor of haptoglobin, zonulin, modulates the permeability of tight junctions (36). Colonic cyclophilin A is secreted in response to inflammatory stimuli such as hypoxia, infection, and other conditions of oxidative stress (37). Another colonic protein with reduced expression, thrombospondin, probably indicates increased angiogenesis (38) and migration of endothelial cells, as well as impaired transforming growth factor $a$-dependent mucosal repair (39).

The observed differences in proteome expression profile between normal and NEC-affected sections in different parts of the gut (intestine, colon) provide new knowledge about the region-specific changes during NEC progression. Proteins related to physiological processes such as antioxidation and carbohydrate, fatty acid, and energy metabolism were clearly region dependent. NEC progression takes quite different molecular pathways in these two regions, probably due to the numerous 
differences in epithelial cell function, local immune system, and bacterial interactions between the small intestine and colon. Although histamine and its receptor appeared similarly regulated in the two regions, antioxidation and heat shock responses to NEC differed. The HSPs are particularly interesting because they have also repeatedly been observed to be affected by NEC in the preterm pig small intestine. HSPs may play different roles at the early and late stages of NEC, which may include managing stress and chaperoning of misfolded proteins. The immunohistochemical pictures of the examined infant intestines revealed that the HSPs were most abundant in cells at the outer layer of villi, perhaps indicating that contact with the luminal microbes may play a role for NEC-related HSP upregulation. The identified proteins related to the heme-homeostasis and nuclear factor- $\mathrm{\kappa B}$ pathway suggest that NEC progression involves heme-centered oxidative stress and inflammatory responses.

The data of the current study could serve as the basis for developing new circulating biomarkers of region-specific NEC development. Unfortunately, we were not able to collect blood samples from the infants investigated in this study, and future investigations should attempt to analyze target-specific protein groups in both plasma and tissue. Secreted proteins are particularly interesting, especially, if they can be located specifically to the small intestine or colon. Further studies are required to show if the circulating levels of specific gut-derived antioxidants, immunoglobulins, antitrypsin, lactoferrin, hemoglobin, and fatty-acid binding protein subunits could serve to identify a region-specific NEC progression during the early phase of the disease, and thus help to support decisions about medical and/or surgical interventions.

\section{METHODS}

\section{Infants and Collection of Intestinal Specimens}

The study was performed on the neonates operated for NEC at Odense University Hospital, Odense, Denmark. Following laparatomy for surgical NEC intervention, necrotic tissue sections $(0.5 \mathrm{~cm})$ and adjacent sections were removed, then either immediately frozen in liquid nitrogen and stored at $-80^{\circ} \mathrm{C}$ for proteomics analyses or fixed in $4 \%$ paraformaldehyde for later histological evaluation. Patient's records were reviewed in order to characterize the clinical findings and disease progression. The study was approved by the ethical committees of the Capital Region of Denmark. All parents gave informed consent to tissue collections. No extra tissue was required for the analyses beyond the tissue removed as a part of the surgical intervention (removing both NEC-affected and adjacent vital sections).

\section{Histology Assessment}

The paraformaldehyde-fixed samples were embedded in paraffin and sectioned at $3 \mu \mathrm{m}$ before being mounted on slides for staining with hematoxylin and eosin. All slides were checked with a light microscope (Orthoplane, Leitz, Germany) and imaged by Image J software (version 1.22c; National Institutes of Health, Bethesda, MD) (40).

\section{Gel-Based Proteomics}

Not all of the selected patients had NEC lesions in both the smallintestine and colon regions, and in some patients the resected NECaffected tissue section in one region was so necrotic that it was considered unsuitable for proteomic analysis. Considering the macroscopic evaluation, small-intestine sections from patients $1,2,4,7,8$, and 9 and colon sections from patients $3,4,5,6,8$, and 10 were used in the proteomic analyses, giving an $n$ value of six for each region $(n=6$ each for NEC-affected and vital small intestine and NEC-affected and vital colon). Extraction of intestinal proteins was carried out as previously described $(6,7)$. Briefly, the tissue specimens were disrupted with a tissue teaser (Biospec Products, Bartlesville, OK) in a cocktail buffer (1\% Triton X-100, $25 \mathrm{mmol} / \mathrm{l} \mathrm{N}$-2-hydroxyethylpiperazine- $\mathrm{N9-2-}$ ethanesufonic acid, $150 \mathrm{mmol} / \mathrm{l} \mathrm{NaCl}, 1 \mathrm{mmol} / \mathrm{l}$ EDTA disodium salt, $1 \mathrm{mmol} / \mathrm{l}$ dithiothreitol, and the protease inhibitor cocktail set III (BioRad)). Superfluous salt in the protein extracts was removed with the trichloroacetic acid-acetone precipitation method as described previously (6-8). The cleaned protein pellets were resuspended in a buffer containing $7 \mathrm{~mol} / \mathrm{l}$ urea, $2 \mathrm{~mol} / \mathrm{l}$ thiourea, $4 \%$ Chaps, and $100 \mathrm{mmol} / \mathrm{l}$ dithiothreitol. Protein concentration was determined with the Protein Quant Kit (GE Healthcare, Piscataway, NJ). The final protein solution was stored at $-80^{\circ} \mathrm{C}$ until proteomic analysis.

The details of the two-dimensional gel electrophoresis procedure have been described previously $(6,7)$. One strip was run on each tissue sample. A protein sample $(100 \mu \mathrm{g})$ was mixed with the rehydration buffer $(9.5 \mathrm{~mol} / \mathrm{l}$ urea, $2 \%$ Chaps, $0.28 \%$ dithiothreitol, $0.5 \%$ immobiline $\mathrm{pH}$ gradient buffer $\mathrm{pI} 3-10$ ) before being added onto one 18-cm ReadyStrip IPG Strip (pI 3-10 Nonlinear, Bio-Rad). The isoelectric focusing was carried out on an Ettan IPGphor III IEF System (GE Healthcare), after a 7 -h active rehydration step, following a five-step program until voltage $\times$ time reached $90,000 \mathrm{Vh}$.

The SDS-PAGE (sodium dodecyl sulfate polyacrylamide gel electrophoresis) of gel strips after isoelectric focusing was carried out using $12.5 \%$ PAGE gel in a Protean xi II cell (Bio-Rad) after a twostep equilibration, with the equilibration buffer $(50 \mathrm{mmol} / \mathrm{l}, \mathrm{pH} 8.8$ Tris-HCl, $6 \mathrm{~mol} / \mathrm{l}$ urea, $30 \%$ glycerol, $2 \%$ SDS) containing $20 \mathrm{mmol} / \mathrm{l}$ dithiothreitol or $20 \mathrm{mmol} / \mathrm{l}$ iodoacetamide. After electrophoresis, the gels were stained with Sypro Ruby Protein Stain (Bio-Rad), then scanned with the Molecular Imager PharosFX Plus System (Bio-Rad), and analyzed with the analyzing software PDQuest 8.0 (Bio-Rad). The matched spots, those that matched among the gels, were assigned numbers. The expression level of matched spots was exported to SPSS 11.5 for statistical analysis.

For identification, protein spots with significant expression difference between groups (necrotic and vital tissue in either small intestine or colon) were cut out manually and subjected to in-gel trypsin digestion as described previously $(6,7)$. The purified protein digests were then subjected to protein identification with mass spectrometry on a 4800 MALDI-TOF/TOF Analyzer (Applied Biosystems, Carlsbad, CA). The protein identity was searched out based on a combination of peptide mass fingerprint and tandem mass spectrometry on a GPS Explorer Workstation (Applied Biosystems) with taxonomy limited to Homo sapiens (human) against the NCBInr database. Basic information of identified proteins was obtained from the mass spectrometry platform, which included protein name, GenInfo identifier, and protein score. A protein identity with a protein score of more than 66 was regarded as correct, considering that the probability of a mismatch in the identification of a protein with a protein score higher than 66 is $P<0.05$.

\section{Western Blot and Immunohistochemistry}

Briefly, $25 \mu \mathrm{g}$ protein was resolved by electrophoresis. Anti-GRP78 and anti-HSP27 (Abcam, Cambridge, UK) were used to detect the expression of HSPA5 and HSP27, respectively. The protein bands were visualized, and the density of the protein bands was detected by Quantity One (Bio-Rad). For histological localization of HSPA5 and HSP27, tissue section slides were deparaffinized and treated with the specific primary antibodies, followed by incubation with a fluorescein isothiocyanate-conjugated secondary antibody to visualize the proteins with microscopy.

\section{Statistics}

All data are expressed as mean \pm SEM. Two-tailed paired $t$-test was used to analyze the difference of protein expression level measured by proteomics or western blot. SPSS 11.5 (SPSS, Somers, NY) was used for all statistical analyses, with $P=0.05$ as the critical level of significance.

\section{ACKNOWLEDGMENTS}

We thank Steffen Husby at the Pediatric Department of University of South Western Denmark, Odense, Denmark; Annie Ravn Pedersen at the National 
Veterinary Institute; and Karen A. Krogfelt of State Serum Institute, Copenhagen, Denmark, for their support. We also thank Kwong-Leung Chan at Queen Mary Hospital, the University of Hong Kong, Hong Kong SAR, China, for his intellectual contribution to this study.

\section{STATEMENT OF FINANCIAL SUPPORT}

This work is supported by the Danish Research Councils.

Disclosure: The authors declared no conflict of interest.

\section{REFERENCES}

1. Obladen M. Necrotizing enterocolitis-150 years of fruitless search for the cause. Neonatology 2009;96:203-10.

2. Hsueh W, Caplan MS, Qu XW, Tan XD, De Plaen IG, Gonzalez-Crussi F. Neonatal necrotizing enterocolitis: clinical considerations and pathogenetic concepts. Pediatr Dev Pathol 2003;6:6-23.

3. Berseth CL. Feeding strategies and necrotizing enterocolitis. Curr Opin Pediatr 2005;17:170-3.

4. Sangild PT, Siggers RH, Schmidt M, et al. Diet-and colonization-dependent intestinal dysfunction predisposes to necrotizing enterocolitis in preterm pigs. Gastroenterology 2006;130:1776-92.

5. Bjornvad CR, Thymann T, Deutz NE, et al. Enteral feeding induces dietdependent mucosal dysfunction, bacterial proliferation, and necrotizing enterocolitis in preterm pigs on parenteral nutrition. Am J Physiol Gastrointest Liver Physiol 2008;295:G1092-103.

6. Jiang P, Sangild PT, Sit WH, et al. Temporal proteomic analysis of intestine developing necrotizing enterocolitis following enteral formula feeding to preterm pigs. J Proteome Res 2009;8:72-81.

7. Jiang P, Siggers JLA, Ngai HH-Y, et al. The small intestine proteome is changed in preterm pigs developing necrotizing enterocolitis in response to formula feeding. J Nutr 2008;60:1491-9.

8. Jiang P, Sangild PT, Siggers RH, Sit WH, Lee CL, Wan JM. Bacterial colonization affects the intestinal proteome of preterm pigs susceptible to necrotizing enterocolitis. Neonatology 2011;99:280-8.

9. Takagaki K, Osawa S, Horio Y, et al. Cytokine responses of intraepithelial lymphocytes are regulated by histamine $\mathrm{H}(2)$ receptor. J Gastroenterol 2009;44:285-96.

10. Schultheiss G, Hennig B, Schunack W, Prinz G, Diener M. Histamineinduced ion secretion across rat distal colon: involvement of histamine $\mathrm{H} 1$ and $\mathrm{H} 2$ receptors. Eur J Pharmacol 2006;546:161-70.

11. Faust D, Raschke K, Hormann S, Milovic V, Stein J. Regulation of alpha1proteinase inhibitor release by proinflammatory cytokines in human intestinal epithelial cells. Clin Exp Immunol 2002;128:279-84.

12. Balch WE, Morimoto RI, Dillin A, Kelly JW. Adapting proteostasis for disease intervention. Science 2008;319:916-9.

13. Shkoda A, Ruiz PA, Daniel H, et al. Interleukin-10 blocked endoplasmic reticulum stress in intestinal epithelial cells: impact on chronic inflammation. Gastroenterology 2007;132:190-207.

14. Werner T, Haller D. Intestinal epithelial cell signalling and chronic inflammation: From the proteome to specific molecular mechanisms. Mutat Res 2007;622:42-57.

15. Patil SB, Bitar KN. RhoA- and PKC-alpha-mediated phosphorylation of MYPT and its association with HSP27 in colonic smooth muscle cells. Am J Physiol Gastrointest Liver Physiol 2006;290:G83-95.

16. Freire F, Romão MJ, Macedo AL, Aveiro SS, Goodfellow BJ, Carvalho AL. Preliminary structural characterization of human SOUL, a haem-binding protein. Acta Crystallogr Sect F Struct Biol Cryst Commun 2009;65(Pt 7):723-6.

17. Sedlak TW, Saleh M, Higginson DS, Paul BD, Juluri KR, Snyder SH. Bilirubin and glutathione have complementary antioxidant and cytoprotective roles. Proc Natl Acad Sci USA 2009;106:5171-6.

18. Nakao A, Otterbein LE, Overhaus M, et al. Biliverdin protects the functional integrity of a transplanted syngeneic small bowel. Gastroenterology 2004;127:595-606.

19. Jiménez A, Pelto-Huikko M, Gustafsson JA, Miranda-Vizuete A. Characterization of human thioredoxin-like-1: potential involvement in the cellular response against glucose deprivation. FEBS Lett 2006;580:960-7.

20. Andersen KM, Madsen L, Prag S, et al. Thioredoxin Txnl1/TRP32 is a redoxactive cofactor of the 26 S proteasome. J Biol Chem 2009;284:15246-54.
21. Chae HZ, Robison K, Poole LB, Church G, Storz G, Rhee SG. Cloning and sequencing of thiol-specific antioxidant from mammalian brain: alkyl hydroperoxide reductase and thiol-specific antioxidant define a large family of antioxidant enzymes. Proc Natl Acad Sci USA 1994;91:7017-21.

22. Sato D, Yanaka A, Shibahara T, et al. Peroxiredoxin I protects gastric mucosa from oxidative injury induced by $H$. pylori infection. J Gastroenterol Hepatol 2008;23:652-9.

23. Ishii $\mathrm{T}$, Itoh $\mathrm{K}, \mathrm{Akasaka} \mathrm{J}$, et al. Induction of murine intestinal and hepatic peroxiredoxin MSP23 by dietary butylated hydroxyanisole. Carcinogenesis 2000;21:1013-6.

24. Naydenov NG, Hopkins AM, Ivanov AI. c-Jun N-terminal kinase mediates disassembly of apical junctions in model intestinal epithelia. Cell Cycle 2009;8:2110-21.

25. Chakravortty D, Nanda Kumar KS. Bacterial lipopolysaccharide induces cytoskeletal rearrangement in small intestinal lamina propria fibroblasts: actin assembly is essential for lipopolysaccharide signaling. Biochim Biophys Acta 2000;1500:125-36.

26. Habtezion A, Toivola DM, Asghar MN, et al. Absence of keratin 8 confers a paradoxical microflora-dependent resistance to apoptosis in the colon. Proc Natl Acad Sci USA 2011;108:1445-50.

27. Cirillo P, Gersch MS, Mu W, et al. Ketohexokinase-dependent metabolism of fructose induces proinflammatory mediators in proximal tubular cells. J Am Soc Nephrol 2009;20:545-53.

28. Schrick JJ, Lingrel JB. cDNA cloning, mapping and expression of the mouse propionyl CoA carboxylase beta (pccb), the gene for human type II propionic acidaemia. Gene 2001;264:147-52.

29. Camarero N, Mascaró C, Mayordomo C, Vilardell F, Haro D, Marrero PF. Ketogenic HMGCS2 Is a c-Myc target gene expressed in differentiated cells of human colonic epithelium and down-regulated in colon cancer. Mol Cancer Res 2006;4:645-53.

30. Snyder CH, Merbitz-Zahradnik T, Link TA, Trumpower BL. Role of the Rieske iron-sulfur protein midpoint potential in the protonmotive Q-cycle mechanism of the cytochrome bc1 complex. J Bioenerg Biomembr 1999;31:235-42.

31. Guthmann F, Börchers T, Wolfrum C, Wustrack T, Bartholomäus S, Spener F. Plasma concentration of intestinal- and liver-FABP in neonates suffering from necrotizing enterocolitis and in healthy preterm neonates. Mol Cell Biochem 2002;239:227-34.

32. Volakakis N, Joodmardi E, Perlmann T. NR4A orphan nuclear receptors influence retinoic acid and docosahexaenoic acid signaling via up-regulation of fatty acid binding protein 5. Biochem Biophys Res Commun 2009;390:1186-91.

33. Kreunin P, Zhao J, Rosser C, Urquidi V, Lubman DM, Goodison S. Bladder cancer associated glycoprotein signatures revealed by urinary proteomic profiling. J Proteome Res 2007;6:2631-9.

34. Tian M, Cui YZ, Song GH, et al. Proteomic analysis identifies MMP-9, DJ-1 and A1BG as overexpressed proteins in pancreatic juice from pancreatic ductal adenocarcinoma patients. BMC Cancer 2008;8:241.

35. Insenser M, Martínez-García MA, Montes R, San-Millán JL, EscobarMorreale HF. Proteomic analysis of plasma in the polycystic ovary syndrome identifies novel markers involved in iron metabolism, acute-phase response, and inflammation. J Clin Endocrinol Metab 2010;95:3863-70

36. Tripathi A, Lammers KM, Goldblum S, et al. Identification of human zonulin, a physiological modulator of tight junctions, as prehaptoglobin- 2 . Proc Natl Acad Sci USA 2009;106:16799-804.

37. Obchoei S, Wongkhan S, Wongkham C, Li M, Yao Q, Chen C. Cyclophilin A: potential functions and therapeutic target for human cancer. Med Sci Monit 2009;15:RA221-32.

38. Danese S. Negative regulators of angiogenesis in inflammatory bowel disease: thrombospondin in the spotlight. Pathobiology 2008;75:22-4.

39. Punekar S, Zak S, Kalter VG, et al. Thrombospondin 1 and its mimetic peptide ABT-510 decrease angiogenesis and inflammation in a murine model of inflammatory bowel disease. Pathobiology 2008;75:9-21.

40. Smith B, Bodé S, Petersen BL, et al. Community analysis of bacteria colonizing intestinal tissue of neonates with necrotizing enterocolitis. BMC Microbiol 2011;11:73. 